\title{
PRECISE SEGMENTATION OF THE LEFT ATRIUM IN C-ARM CT VOLUMES WITH APPLICATIONS TO ATRIAL FIBRILLATION ABLATION
}

\author{
Yefeng Zheng ${ }^{\star} \quad$ Matthias John ${ }^{\dagger} \quad$ Gareth Funka-Lea ${ }^{\star} \quad$ Jan Boese ${ }^{\dagger} \quad$ Dorin Comaniciu \\ * Image Analytics and Informatics, Siemens Corporate Research, USA \\ ${ }^{\dagger}$ Healthcare Sector, Siemens AG, Germany
}

\begin{abstract}
A patient-specific left atrium (LA) model extracted from intra-operative C-arm CT plays an important role in surgical planning for transcatheter left atrial fibrillation ablation. Overlaying the LA model onto 2D fluoroscopic images provides valuable visual guidance during surgery. However, automatic segmentation of the LA, together with the left atrial appendage (LAA) and the pulmonary vein (PV) trunks, is challenging due to the large structural variations and nonoptimal image quality. In this paper, we exploit a part based LA model to handle the structural variations and different parts are then merged into a consolidated mesh. The connection region between the PV/LAA and the LA chamber is segmented precisely by enforcing both image boundary delineation accuracy and mesh smoothness. Furthermore, the boundary between parts is optimized to improve the mesh part labeling accuracy.
\end{abstract}

Index Terms - Left Atrium Segmentation, Fibrillation Ablation, C-arm CT

\section{INTRODUCTION}

About $15 \%$ of all strokes (which are the third leading cause of death in the USA) are caused by atrial fibrillation (AF).As a widely used minimally invasive surgery to treat $A F$, the catheter based ablation procedure uses high radio-frequency energy to eliminate the sources of ectopic foci, especially around the pulmonary venous (PV) ostia. Automatic segmentation of the left atrium (LA) is important in pre-operative assessment to identify the potential sources of the abnormal electrical events $[1,2]$. However, there are large variations in the PV drainage patterns [3] with the most common variations (about $20-30 \%$ of the whole population) as extra right PVs and the left common PV (where the two left PVs merge into one before joining the chamber). A personalized LA model can help to translate a generic ablation strategy to the specific patient's anatomy, thus making the ablation strategy more effective for this patient. It can also be overlaid onto $2 \mathrm{D}$ realtime fluoroscopic images to provide visual guidance during surgery.
Recently, C-arm computed tomography (CT) is emerged as a new imaging technique to provide $3 \mathrm{D}$ model of the LA. Compared to conventional CT or magnetic resonance imaging (MRI), the advantage of C-arm CT is that overlay of the 3D patient-specific LA model onto a 2D fluoroscopic image is straightforward and accurate since both $3 \mathrm{D}$ and $2 \mathrm{D}$ images are captured on the same device within a short time interval. Normally, a non-electrocardiography-gated acquisition is performed to reconstruct a C-arm CT volume, therefore, it contains severe cardiac motion artifacts. For a $\mathrm{C}$-arm with a small X-ray detector panel, part of the body may be missing in some 2D X-ray projections due to the limited field-of-view, resulting in significant artifacts around the margin of a reconstructed volume. In addition, there may be severe streak artifacts caused by various interventional devices inserted in the body.

Various methods have been proposed for LA segmentation and they can be roughly categorized as non-model based or model based approaches. The non-model based approaches $[1,2]$ do not assume any prior knowledge of the LA shape and the whole segmentation procedure is purely data driven. The advantage of these methods is that they can handle structural variations of the PVs. However, such methods cannot provide the underlying anatomical information (e.g., which part of the segmentation is the left inferior PV). In practice, non-model based approaches work well on CT and MRI datasets, but they are not robust on challenging $\mathrm{C}$-arm CT. The model based approaches exploit a prior shape of the LA (either in the form of an atlas $[4,5]$ or a mean shape mesh [6]) to guide the segmentation. With a prior shape constraint, they could avoid the leakage around weak or missing boundaries, which plagues the non-model based approaches. However, it is difficult to handle the structural variations, e.g., the left common PV, using a holistic shape model [6]. The $\mathrm{PV}$ variations were addressed in [5] using multiple atlases, at the cost of extra computation time.

Recently, we proposed a fully automatic part based LA segmentation system on C-arm CT [7]. Instead of using one mean shape model [6], the challenge of PV structural variations is addressed using a part based model, where the whole LA is split into the chamber, appendage, and four major PVs. Each part is a much simpler anatomical structure compared to 
the holistic one, therefore can be detected and segmented using a model based approach. Though each part is segmented well, the connection region to the LA chamber (i.e., the region around the ostia of the PVs and appendage) is difficult to segment precisely. In atrial fibrillation ablation, tissues around the PV ostia are the main focus of ablation. Therefore, the segmentation accuracy around the PV ostia is far more important than other non-ablation regions. In this work, we propose a method for precise segmentation of the ostia region by enforcing both image boundary delineation accuracy and mesh smoothness. A novel approach is also proposed to improve the labeling of the mesh parts (determining the exact boundary between the appendage/PVs and LA chamber).

\section{MULTI-PART LEFT ATRIUM SEGMENTATION}

Our part based LA model includes the LA chamber body, appendage, and four major PVs. The shape of the appendage is close to a tilted cone and the PVs are tubular structures. For AF ablation, physicians only care about a proximal PV trunk, therefore, we only detect a trunk of $20 \mathrm{~mm}$ in length, originating from its ostium. Each LA part is a much simpler anatomical structure compared to the holistic one, therefore can be detected and segmented using a model based approach. We use marginal space learning (MSL) [8] to detect and segment each part. MSL is an efficient method to estimate the position, orientation, and size of an object in a 3D volume. After automatic object pose estimation, a mean shape is aligned with the pose as an initial mesh. A machine learning based boundary detector [8] is used to guide the boundary evolution. For each LA part, we have an MSL based pose detector and a learning based boundary detector. Interested readers are referred to [8] for more details of MSL. After detecting and segmenting all parts, we merge them into a consolidated mesh, which is the main topic of this paper.

Compared to the holistic approach [6], the part based approach can handle large structural variations. The MSL based detection/segmentation works well for big anatomical structures, e.g., the LA chamber. However, independent detection of other parts is not robust, either due to the low contrast (appendage) or small object size (PVs). In [7], we proposed a method to enforce statistical shape constraint during the pose estimation of PVs to improve the detection robustness.

\section{PRECISE SEGMENTATION OF OSTIA REGION OF PULMONARY VEINS AND APPENDAGE}

In this section, we present our algorithm for precise segmentation of the ostia region of the PVs and appendage. The system diagram is shown in Fig. 1. After shape-constrained detection and segmentation, we get six separate meshes (the LA chamber, appendage, and four PVs). There may be gaps and/or intersections among different meshes. Physicians prefer a consolidated mesh with different anatomical structures

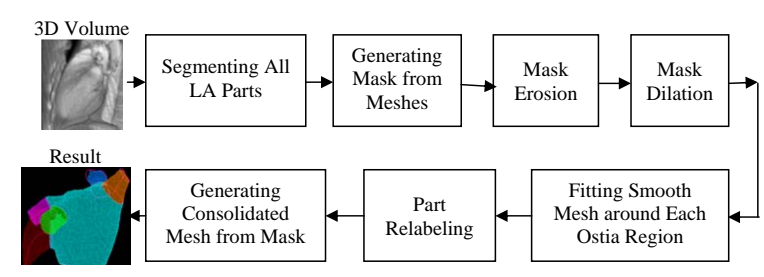

Fig. 1. Diagram of the proposed LA segmentation method.

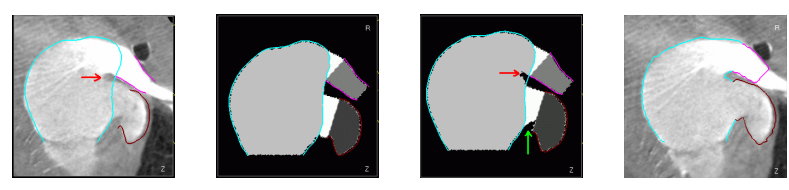

Fig. 2. Refinement of the segmentation of the ridge between PV and appendage. Left: An planar cut of a volume overlaid with the LA part meshes with cyan for the chamber, dark red for the appendage, and magenta for the left superior PV. Left Middle: Mask after connecting the PV/appendage meshes to the LA chamber. The initial ostia region is shown in white. Right Middle: Mask after erosion. Right: Final mesh.

labeled with different colors. We first project the proximal ring of a PV or appendage (which is defined as the rim of a tubular mesh close to the LA chamber) along the tube centerline onto the LA chamber to eliminate the gaps among different mesh parts. Now, the part meshes are fully connected. However, the mesh intersections of different parts may still be present. It is complicated to work directly on the meshes. Instead, we convert the meshes to a volume mask, and generate a new mesh from the volume mask using the marching cubes algorithm [9]. Since pure mesh operation is performed to connect PV/appendage meshes to the LA chamber, the ostia region is not segmented accurately and needs to be further refined using the approaches detailed in the following.

Mask Refinement: The ridge between the left superior PV (LSPV) and the appendage is often not delineated accurately, especially when the LSPV and the appendage are close to each other. As shown in Fig. 2, a narrow ridge is partially enclosed inside the LA chamber mesh. To refine the segmentation around the ridge, we perform layer-by-layer erosion to remove dark voxels. We first find the outer layer of the chamber and ostia regions. If a voxel on the outer layer has an intensity less than a threshold, we set it to a background voxel. Such layer-by-layer erosion is performed 10 iterations. This erosion operation not only improves the segmentation of the ridge, but also other regions. For example, as shown in Fig. 2, the dark voxels around the appendage ostium are removed, resulting in more accurate segmentation around that region too. A C-arm CT normally has a high intensity variation due to the lack of a standard protocol for the use of contrast agent. A fixed erosion threshold does not work for all datasets. Instead, we automatically determine an adaptive threshold for 

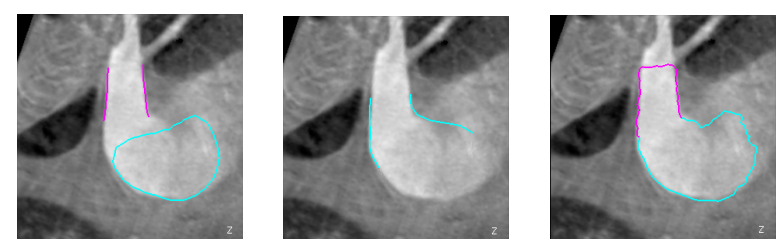

Fig. 3. Segmenting the ostia region of a pulmonary vein. Left: Separated part meshes. Middle: Fitting a smooth mesh onto the ostia region. Right: Final mesh.
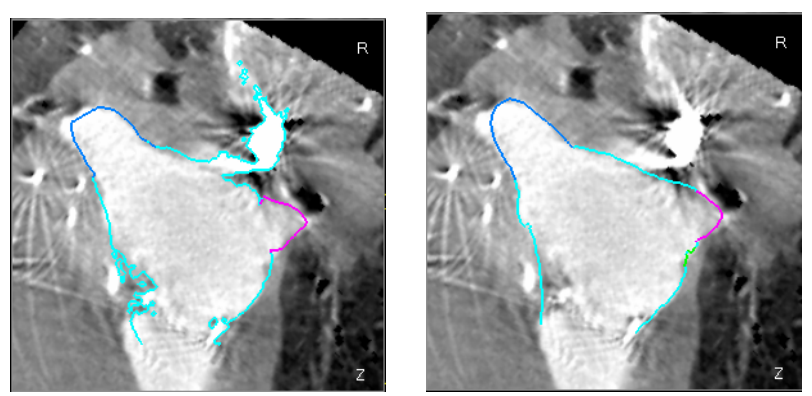

Fig. 4. Avoid segmentation leakage. Left: Segmentation after region growing. Right: Segmentation after enforcing smoothness of the ostia region.

each dataset based on the analysis of the ostia region intensity. To be specific, we sort the intensity of the ostia region and use the lower $2.5^{\text {th }}$ percentile as the erosion threshold. Experiments show that this parameter setting results in good segmentation.

The initial ostia region is labeled with pure mesh operation by projecting the PV/appendage meshes onto the chamber. The segmentation is often not accurate when the part gap is too large. After erosion, we perform layer-by-layer region growing to refine the segmentation using an adaptive threshold. A conservative growing threshold is set (i.e., the $50^{\text {th }}$ percentile of the initial ostia region intensity) to include only very bright voxels.

Due to severe noise in $\mathrm{C}$-arm CT, the resulting boundary after region growing may be quite zig-zag. On very rare cases, it may leak into other neighboring high-contrasted objects (e.g., the trans-esophagus echocardiographic (TEE) probe in Fig. 4). To fix these issues, we fit a smooth mesh in the ostia region. The mesh is initialized as a tube. We extend the proximal ring of a PV (or appendage) towards the chamber. We then triangulated the surface between the original proximal ring and the extended ring as an initial mesh. Each mesh point is moved along the surface normal to the first transition from a masked voxel to background. Many leaked voxels are already excluded from the adjusted mesh. However, the resulting mesh is not smooth and it may still include minor leakage. Finally, we search for an optimal smooth mesh by the shrinking the mesh points along the surface normal.

A smooth mesh is fit for each ostia region (in total of

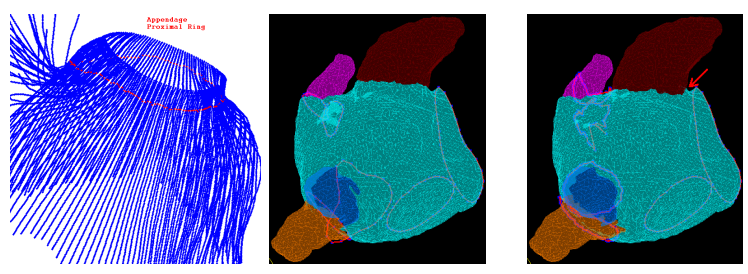

Fig. 5. Mesh part relabeling. Left: Optimal boundary (red contour) between the left atrial appendage and chamber. Middle: Before mesh part relabeling. Right: After relabeling.

five) and an ostia region voxel is included in the final mask if and only if it is inside an ostia mesh. After fitting an optimal smooth mesh around the ostia region, we can achieve a smooth mesh in the final segmentation and at the same time prevent leakage (as shown in Fig. 4).

Mesh Part Labeling: Besides precise segmentation of the ostia region, the mesh parts should also be labeled accurately. Normally, the boundary between different parts has high surface curvature on the mesh. However, sometimes, the connection can be smooth, therefore, the exact boundary around those regions need to be constrained by the neighboring high-curvature regions. We propose an optimization based approach to search for the part boundary. First the proximal ring of a PV is densely resampled to $0.5 \mathrm{~mm}$. Suppose the proximal ring center is $C$. Given a proximal ring point $P_{i}$, a plane is determined that is perpendicular to the proximal ring plane, and passes $P_{i}$ and $C$. Starting from point $P_{i}$, we trace the mask boundary (the boundary between foreground voxels and the background) on the plane. The tracing stops if it encounters a masked voxel of another PV (or appendage) or the total traced length is more than $60 \mathrm{~mm}$. The traced contour is then uniformly resampled to a high resolution (e.g., $0.25 \mathrm{~mm}$ ). Left sub-figure of Fig. 5 shows the traced contours from the appendage proximal ring. This procedure generates a set of points $Q_{i}^{j}$. Here, $Q_{i}^{j}$ means the $j^{\text {th }}$ point on the $i^{\text {th }}$ contour and $Q_{i}^{0}=P_{i}$. We then search for a smooth part boundary $B$ with the maximum sum of curvature,

$$
B=\left(Q_{0}^{J(0)}, Q_{1}^{J(1)}, \ldots, Q_{n-1}^{J(n-1)}\right)=\underset{J(0), J(1), \ldots, J(n-1)}{\arg \max } \sum_{i=0}^{n-1} C\left(Q_{i}^{J(i)}\right) .
$$

Here, $C\left(Q_{i}^{j}\right)$ is the curvature at point $Q_{i}^{j}$, which is approximated as

$$
C\left(Q_{i}^{j}\right)=\left\|\left(Q_{i}^{j+1}-Q_{i}^{j}\right)-\left(Q_{i}^{j}-Q_{i}^{j-1}\right)\right\|=\left\|2 Q_{i}^{j}-Q_{i}^{j-1}-Q_{i}^{j+1}\right\| . \quad(2)
$$

As a second-order derivative of a contour, curvature estimation is error prone, therefore, for some datasets, the final part boundary $B$ may be a bit away from the chamber. To improve robustness, we add a small amount of bias, $\left\|N .\left(Q_{i}^{j}-Q_{i}^{0}\right)\right\|$ in the cost function to push the part boundary toward the chamber. Here, $N$ is the normal of the proximal ring and $N .\left(Q_{i}^{j}-Q_{i}^{0}\right)$ is the dot-product of vectors $N$ and $Q_{i}^{j}-Q_{i}^{0}$, therefore measuring the distance from $Q_{i}^{j}$ to the proximal ring plane. We enforce the smoothness of the part boundary by constraining the distance of neighboring $Q_{i}^{J(i)}$ and $Q_{i+1}^{J(i+1)}$ to $\|J(i)-J(i+1)\| \leq 1$. The part boundary should form a closed contour, therefore $\|J(n-1)-J(0)\| \leq 1$. The final 
Table 1. Left atrium segmentation errors (based on fourfold cross validation) on C-arm CT datasets with 253 large volumes and 434 small volumes. The symmetric surface-tosurface errors, measured in millimeters $(\mathrm{mm})$, are reported.

\begin{tabular}{|c|c|c|c|c|}
\hline \multirow{2}{*}{} & \multicolumn{2}{|c|}{ Large Volumes } & \multicolumn{2}{c|}{ Small Volumes } \\
\cline { 2 - 5 } & Mean & Median & Mean & Median \\
\hline \hline LA Chamber & 1.61 & 1.41 & 1.62 & 1.44 \\
\hline Appendage & 2.87 & 2.20 & 2.69 & 2.21 \\
\hline Left Inf. PV & 1.81 & 1.58 & 1.96 & 1.43 \\
\hline Left Sup. PV & 1.78 & 1.44 & 1.69 & 1.16 \\
\hline Right Inf. PV & 1.80 & 1.42 & 1.83 & 1.52 \\
\hline Right Sup. PV & 1.52 & 1.39 & 1.62 & 1.27 \\
\hline
\end{tabular}

optimization problem is formalized as

$B=\left(Q_{0}^{J(0)}, \ldots, Q_{n-1}^{J(n-1)}\right)=\arg \max \sum_{i=0}^{n-1}\left[C\left(Q_{i}^{J(i)}\right)+w\left\|N \cdot\left(Q_{i}^{J(i)}-Q_{i}^{0}\right)\right\|\right]$.

Here $w$ is a weight adjusting the bias toward a boundary close to the chamber. Throughout our experiments, we set $w=0.001$. The optimal part boundary can be solved efficiently using dynamic programming to achieve a global optimal solution. Fig. 5 shows the mesh before and after part relabeling. The appendage mesh is successfully extended toward the chamber in this example.

\section{EXPERIMENTS}

We collected 687 C-arm CT datasets, scanned by Siemens Axiom Artis zee $\mathrm{C}$-arm systems at 17 clinical sites from Europe and the USA. Among them, 253 datasets were scanned with large X-ray detector panels $\left(30 \times 40 \mathrm{~cm}^{2}\right)$ with an isotropic volume resolution of $0.30 \mathrm{~mm}^{3}$. The other 434 datasets were scanned with small X-ray detectors $(20 \times 20$ $\mathrm{cm}^{2}$ ) with a volume resolution of $0.18 \mathrm{~mm}^{3}$. Due to the limited field-of-view of small X-ray detectors, the reconstructed volumes may contain significant artifacts, especially around the volume margin. We train two separate systems for the large and small volumes, respectively.

A four-fold cross validation is performed to evaluate our algorithm and the segmentation accuracy is measured using the symmetric surface-to-surface distance. Table 1 shows segmentation errors of the consolidated meshes. For a mesh part, we measure the distance to the corresponding part in the ground truth, therefore both the segmentation and mesh part labeling errors are penalized. The mean errors of the PV and LA chamber range from 1.61 to $1.96 \mathrm{~mm}$, while the error of the appendage is significantly larger due to the weak contrast inside the appendage. We cannot directly compare our segmentation accuracy with those reported in the literature due to the difference in imaging modalities, datasets, and LA models. There is no quantitative evaluation available in $[1,2]$. To the best of our knowledge, there is only one work [6] reporting a slightly smaller error, however, their test set of 33 patients is significantly smaller than ours.

The proposed method is computationally efficient, taking about $2.3 \mathrm{~s}$ (on a computer with quad-core $2.33 \mathrm{GHz}$ CPUs) to process a volume with $256 \times 256 \times 250$ voxels. It compares favorably with the previous methods, e.g., $5 \mathrm{~s}$ in [4], 5-45 s in [1], $30 \mathrm{~s}$ in [6], and more than two hours in [5].

\section{CONCLUSION}

In the work, we presented a part based LA segmentation method, which can handle structural variations elegantly. Post-processing is performed to further improve the segmentation accuracy of the LSPV-appendage ridge and the ostia region of the PVs and appendage. Furthermore, we also improved the labeling of mesh part boundary on the final consolidated mesh using an optimization based approach.

\section{REFERENCES}

[1] M. John and N. Rahn, "Automatic left atrium segmentation by cutting the blood pool at narrowings," in MICCAI, 2005, pp. 798-805.

[2] R. Karim, R. Mohiaddin, and D. Rueckert, "Left atrium segmentation for atrial fibrillation ablation," in Proc. of SPIE Medical Imaging, 2008.

[3] E. M. Marom, J. E. Herndon, Y. K. Kim, and H. P. McAdams, "Variations in pulmonary venous drainage to the left atrium: Implications for radiofrequency ablation," Radiology, vol. 230, pp. 824-829, 2004.

[4] R. Karim, C. Juli, L. M. Lawes, P. Kanangaratnam, D. W. Davies, N. S. Peters, and D. Rueckert, "Automatic segmentation of left atrial geometry from contrast-enhanced magnetic resonance images using a probabilistic atlas," in MICCAI Workshop on STACOM, 2010, pp. 134-143.

[5] M. Depa, M. R. Sabuncu, G. Holmvang, R. Nezafat, E. J. Schmidt, and P. Golland, "Robust atlas-based segmentation of highly variable anatomy: Left atrium segmentation," in MICCAI Workshop on STACOM, 2010, pp. 85-94.

[6] R. Manzke, C. Meyer, O. Ecabert, J. Peters, N. J. Noordhoek, A. Thiagalingam, V. Y. Reddy, R. C. Chan, and J. Weese, "Automatic segmentation of rotational X-ray images for anatomic intra-procedural surface generation in atrial fibrillation ablation procedures," IEEE Trans. Medical Imaging, vol. 29, no. 2, pp. 260-272, 2010.

[7] Y. Zheng, T. Wang, M. John, S. K. Zhou, J. Boese, and D. Comaniciu, "Multi-part left atrium modeling and segmentation in C-arm CT volumes for atrial fibrillation ablation," in MICCAI, 2011, pp. 487-495.

[8] Y. Zheng, A. Barbu, B. Georgescu, M. Scheuering, and D. Comaniciu, "Four-chamber heart modeling and automatic segmentation for 3D cardiac CT volumes using marginal space learning and steerable features," IEEE Trans. Medical Imaging, vol. 27, no. 11, pp. 1668-1681, 2008.

[9] W. E. Lorensen and H. E. Cline, "Marching cubes: A high resolution 3D surface construction algorithm," Computer Graphics, vol. 21, no. 4, pp. 163-169, 1987. 